\title{
4D flow characterization of aortic blood flow after valve sparing root reimplantation procedure
}

\author{
Nicola Galea ${ }^{1,2}$, Filippo Piatti ${ }^{3}$, Christopher Lau $^{4}$, Francesco Sturla ${ }^{3,5}$, Luca Weltert ${ }^{6}$, Iacopo Carbone ${ }^{2}$, \\ Ruggero De Paulis ${ }^{6}$, Mario Gaudino ${ }^{4}$, Leonard N. Girardi ${ }^{4}$; The Cornell International Consortium for \\ Aortic Surgery (CICAS)
}

${ }^{1}$ Department of Experimental Medicine, ${ }^{2}$ Department of Radiological, Oncological and Pathological Sciences, Sapienza University of Rome, Rome, Italy; ${ }^{3}$ Department of Electronics, Information and Bioengineering, Politecnico di Milano, Milan, Italy; ${ }^{4}$ Department of Cardiothoracic Surgery, Weill Cornell Medicine, New York, USA; 3 D and Computer Simulation Laboratory, IRCCS Policlinico San Donato, San Donato Milanese, Italy; ${ }^{6}$ Department of Cardiac Surgery, European Hospital, Rome, Italy

Correspondence to: Leonard N. Girardi, MD. Department of Cardiothoracic Surgery, Weill Cornell Medicine, 525 E 68th St, M-404, NY 10065, USA. Email: lngirard@med.cornell.edu.

\begin{abstract}
Valve-sparing aortic root replacement (VSRR) with reimplantation technique is an effective alternative for young patients with dilated roots and preserved cusps, which avoids the risks of lifelong anticoagulation or valve degeneration. New grafts with anatomically-shaped sinuses have been developed in order to preserve aortic root physiology, which could decrease complication rates and improve durability. However, controversy remains regarding the effect of recreation of the sinuses of Valsalva during VSRR on long-term outcomes. The novel 4D flow technique, exploiting its unique ability to combine anatomical evaluation of the root with fluid-dynamic assessment of aortic flow, enables integrated analysis of the close interaction between graft design, valvular morphology and three-dimensional (3D) flow characteristics. Early experimental studies have shown how graft shape affects the aortic root flow pattern, formation of vortexes and helicity of downstream flow; however, the clinical significance of these findings is yet to be clarified. Various and still unexplored knowledge can be obtained from the qualitative and quantitative analysis of these complex datasets, that could shed more light on which is the best among myriad surgical techniques and grafts adopted in VSRR. The extraordinary potential 4D flow imaging opens new boundless horizons in the perspective of an increasingly patient-tailored surgical planning.
\end{abstract}

Keywords: Aortic root replacement; valve-sparing aortic root replacement (VSRR); neo-sinuses graft; vortex; sinuses of valsalva; 4D flow imaging

Received: 01 January 2018; Accepted: 15 March 2018; Published: 09 May 2018.

doi: 10.21037 /jovs.2018.03.17

View this article at: http://dx.doi.org/10.21037/jovs.2018.03.17

\section{Introduction}

Valve-sparing aortic root replacement (VSRR), utilizing the "reimplantation technique", is a comprehensive surgical procedure for the treatment of aortic root aneurysms with intact aortic valve cusps, which allows patients to avoid the need for lifelong anticoagulation therapy or future re-operation associated with prosthetic valves. Beyond examining the clinical outcomes, there now exists the possibility of studying aortic flow dynamics in vivo using novel advanced flow-sensitive magnetic resonance imaging (MRI) techniques, which have opened new horizons for a better and deeper understanding of the interaction between anatomical surgical results and post-operative flow dynamics.

Indeed, 4D flow MRI has the unique capability of combining into a "one stop shop", the simultaneous assessment of aortic morphology with the evaluation of three-dimensional (3D) geometric flow patterns, characteristics of turbulent kinetic energy and central 

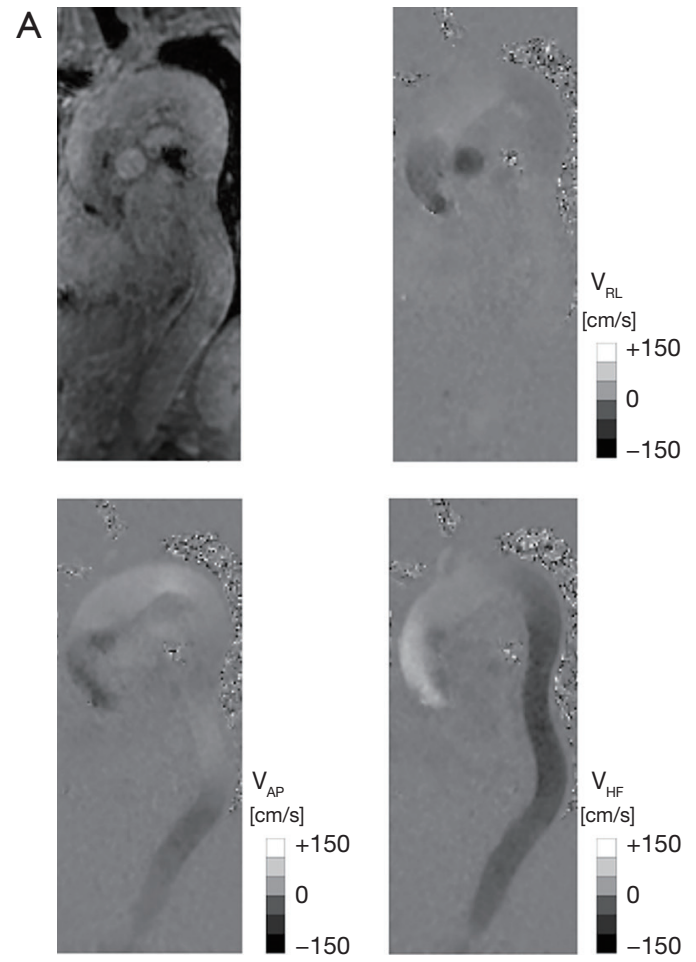

B

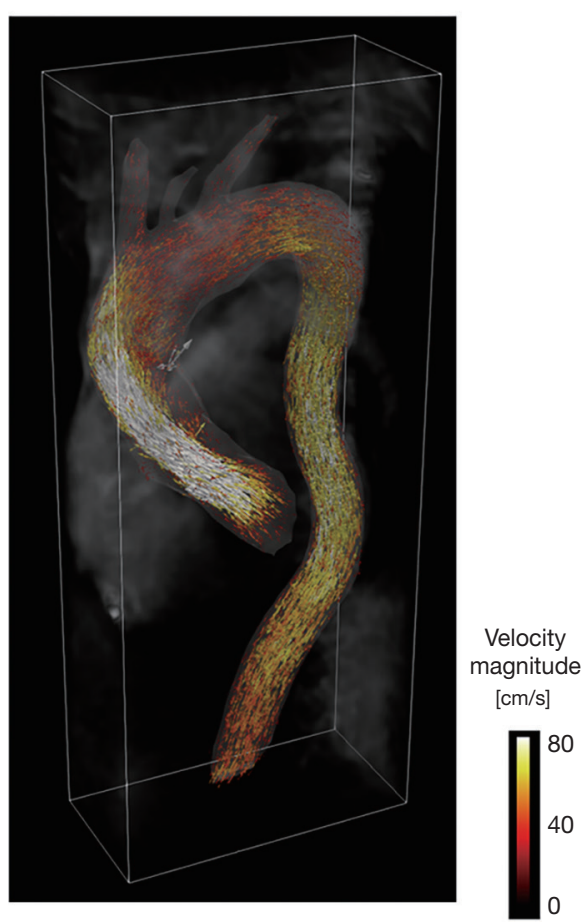

Figure $14 \mathrm{D}$ flow acquired and post-processed images. (A) Representation on a single sagittal slice of the information yielded by 4D flow MRI, i.e., anatomical and velocity along the right-left $\left(V_{\mathrm{RL}}\right)$, antero-posterior $\left(\mathrm{V}_{\mathrm{AP}}\right)$ and head-foot $\left(\mathrm{V}_{\mathrm{HF}}\right)$ directions; (B) volume rendering superimposed to $3 \mathrm{D}$ vectors of the velocity field color-coded by velocity magnitude. MRI, magnetic resonance imaging.

laminar flow components, and interactions between the flowrelated pressure fields and elastic response of the aortic wall.

\section{D flow MRI}

MRI inherently exhibits sensitivity to tissue motion and therefore to fluid flow, thus allowing for the design of adhoc pulse sequences with bipolar flow encoding gradients to generate a velocity sensitive MRI signal phase, which can be used to directly quantify blood flow velocities (1). This technique, known as phase contrast MRI (PCMRI), has become a diagnostic tool for the quantification of pulsatile blood flow in the heart and large vessels on selected 2D planes, measuring standard flow parameters, such as peak velocity, net flow, stroke volume, regurgitant fraction, and shunt flows in congenital diseases (1). However, the complexity of the heart and cardiovascular system, combined with the intrinsic three-dimensionality and multi-directionality of blood flow during the cardiac cycle, raised the necessity of measuring $3 \mathrm{D}$ volumetric time-resolved flow fields. To do so, the concept of 2D PC-
MRI was extended to design 3D PC-MRI pulse sequences with three-directional velocity encoding, namely "4D flow MRI" sequences (2) (Figure 1). During the last few decades, 4D flow MRI has allowed analysis of in vivo flow fields within the cardiovascular system in a more comprehensive way than was previously possible, offering the possibility to retrospectively analyze any location of interest within the acquired volume and to extract complex hemodynamic markers thanks to the full three-directional velocity field coverage (2).

In particular, 4D flow MRI has been applied to measure in vivo blood flow in the great arteries of the heart, e.g., pulmonary artery and aorta, where the combination of standard-of-care MRI (e.g., cineMR, angiography) with advanced flow imaging provides a comprehensive assessment of aortic/pulmonary structure and function (2). Various visualization tools were exploited to investigate the spatial and temporal blood flow patterns in the aortas of healthy subjects, thus revealing the presence of righthanded helical flow after the accelerated passage of blood through the aortic valve plane, as well as late systolic 
retrograde flow which contributes to coronary perfusion (3). The alterations of these flow patterns have been of major interest in the last decade, as potential causes of various diseases affecting the aorta as well as congenital or acquired aortic geometry aberrations or valvular diseases, which may result in substantially altered aortic hemodynamics $(2,4)$.

\section{Valve sparing aortic root reimplantation}

Up to the early nineties the very concept of replacing the aortic root while simultaneously sparing the aortic valve did not exist. The pioneering work of Sir Maghdi Yacoub in remodeling of the aortic bulb with a Dacron graft succeeded (5) in preserving the native valve but it remained mostly anecdotal experience, with little standardization.

This probably was due to the intrinsic configuration of the aortic valve, which has a $3 \mathrm{D}$ base, with commissures rising up almost to the level of the sinotubular junction, making preservation of anatomic relations difficult when resecting the sinuses.

Then Tirone David brilliantly devised a method to excise the aneurysmal aortic sinus tissue and reimplant the valve inside a Dacron tube, securing it at the annular level on a single plane, and resuspending the commissures inside of tube. The "reimplantation" procedure was born, commonly called the "David" procedure (6).

Initially confined to a selected group of patients, the procedure was gradually adopted to treat aortic roots with good quality leaflets, and results proved consistent among different centres worldwide. While an increasing number of studies have validated the excellent clinical outcome of this procedure, little data exist about the impact on aortic fluiddynamics. In particular, few studies have examined patients repaired with straight tube grafts using 4D flow MRI (3,7-10).

Controversial results arose from investigations on the postoperative normalization of aortic flow within the neoroot. Markl et al. (3) affirmed the readily detectable systolic vortex formation in all patients after VSRR behind all coronary cusps, whether or not graft neo-sinuses were created, with no significant differences in degree of vorticity with visual grading ( 0 , none; $1-3$, minimal-prominent) compared to volunteers. They postulated that vortex formation is not predominantly a consequence of graft shape but is more caused by hemodynamic properties of aortic blood flow (e.g., pressure differences across the aortic cusps).

Conversely, other studies reported highly disturbed flow and small re-circulating vortices adjacent to the straight graft wall, supporting the evidence that, the relative lack of space between the open valve leaflets and aortic root wall does not allow proper vortex formation $(7,9,11)$ (Figure $2 A, B)$.

Oechtering (9) et al. found small sinus vortices malrotated 90 degrees along the vessel axis in three patients after reimplantation of a straight aortic graft compared to the volunteer's normal vortex.

In our experience, among eight patients who received reimplantation with straight graft examined by 4D flow MRI, nobody exhibited supra-valvular vortex generation (12). In particular, the turbulent flow detectable in the proximal ascending aorta was inconstant, disorganized, asymmetric, and did not respect any rotational axis (12).

Further investigation utilizing 4D flow MRI could clarify the relationships between the generation of these irregular turbulent flows, the echocardiographic evidence of systolic contact of the valve leaflets with the graft wall (13) and the characteristics of compliance related to the elastic response of the Dacron graft.

Furthermore, it remains to be elucidated whether this turbulent flow may increase the stress load on the graft wall or valve leaflets and to explore the connection between specific flow patterns and risk of complications.

In order to investigate the potential role of 4D Flow MRI in predicting complications, Hope et al. (8) correlated the aortic flow pattern of 12 Marfan patients after VSRR and examined adverse outcomes, such as aneurysm formation and dissection over 5-year of follow-up. One patient developed a Stanford Type B aortic dissection. Altered flow patterns and hemodynamic stresses were retrospectively present in the thoracic aorta (altered peak systolic flow patterns with vertically oriented vortex in the ascending aorta and in proximal descending aorta, altered wall shear stress and decreased average velocities in the region of the dissection). The relationship between flow patterns and the subsequent dissection remains to be determined.

\section{Modified graft with reconstruction of the sinuses of Valsalva}

With widespread adaptation of the reimplantation technique, one anatomical aspect remained neglected: the native aortic root was bulb-shaped, while the graft was a straight cylinder. As there was no apparent clinical significance in the original reimplantation cohort, the concern was put aside. Moreover, no practical technology was yet available to produce a bulbshaped prosthesis. The importance of the sinuses of Valsalva in the generation of vortices and the function of the aortic valve was theorized since the time of Leonardo da Vinci (14) 
A

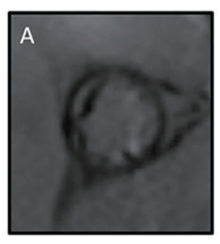

C

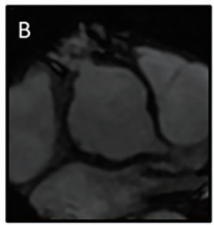

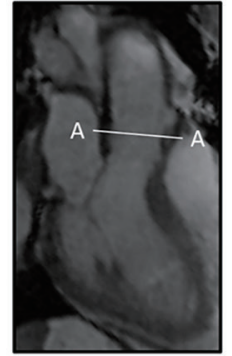

B

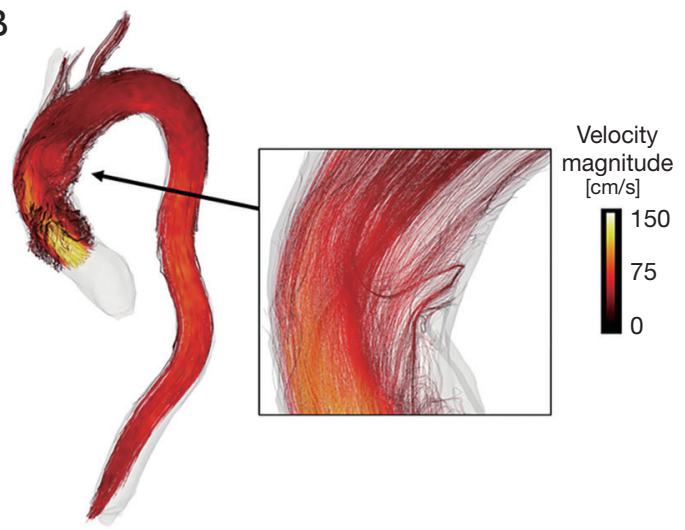

D

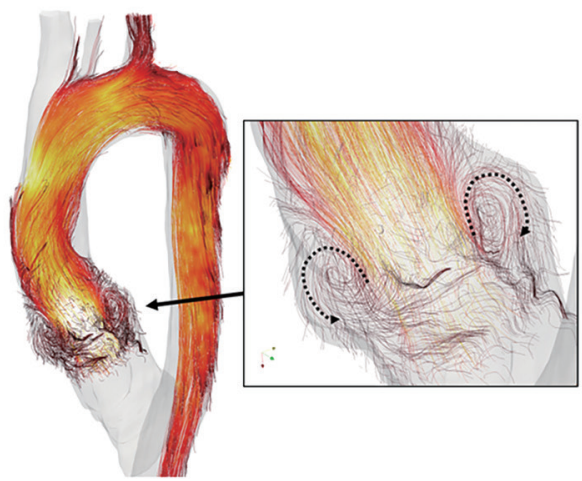

Figure 2 4D Flow MRI images of two patients implanted with a straight tube graft $(\mathrm{A}, \mathrm{B})$ and a graft with reconstruction of neo-sinuses $(C, D)$. CineMR images (A,C) have been acquired on aortic root short axis (left) and long axis (right), in order to depict aortic root anatomy. 3D streamlines describing the bulk flow field at peak systole for two patients implanted with a straight tube graft (B) and a graft with reconstruction of neo-sinuses (D). Streamlines are color-coded based on the instantaneous and local 3D velocity magnitude. The enlarged boxes show the disorganized turbulent flow within straight tubular graft (B) and the vortices of the graft with reconstruction of neo-sinuses (dotted curved arrows in the region of coronary sinuses of Valsalva, D). MRI, magnetic resonance imaging.

and was first experimentally explored by Bellhouse and Bellhouse in 1968 (15). In the early two thousands, an Italian Surgeon, Ruggero De Paulis, created an elegant and effective solution. Observing that a Dacron graft expands only on the axis of the corrugations, he cut the base of the straight tube, rotated it 90 degrees, and reattached it. This resulted in corrugations that were aligned on an orthogonal plane at the base, re-creating the bulbar shape of the aortic root. With low manufacturing impact and a diameter 30\% larger than the straight graft, roughly the same as the average native sinuses of Valsalva: the Valsalva Prosthesis was born (16).

After initial doubts the surgical community embraced the technology at increasing rates. Opening and closing patterns in pulse replicator environments (17), finite elements stress strain analysis (18) and clinical follow up studies (19-21) all converged at demonstrating adequate structural integrity with the new design. With non-inferiority granted, continued design improvements ensued and prefabricated grafts became available.

Physiologically, the presence of a sinotubular ridge and the rounded shape of sinuses of Valsalva promotes vortex formation during late systole, by initiating the early and smooth closure of the aortic valve (15) and redistributing the load and stress related to retrograde aortic flow evenly on the valve leaflets and the aortic wall.

4D flow imaging enabled us to visualize the vortex in vivo and demonstrated that the rotational direction of the vortex is from superior to inferior along the external margins of the sinuses and then turns toward the central aortic orifice at the base of the root, by exerting an inward pressure on 
the aortic leaflets and facilitating rapid valve closure at endsystole (7) (Figure 2C,D).

However, the effects of recreating neo-sinuses of Valsalva on aortic fluid-dynamics during VSRR is still debated and a matter of great interest.

Oechtering et al. (22) visualized sinus vortices by streamlines and time-resolved particle paths using 4D flow MRI in twelve patients with a modified graft with sinus reconstruction (Uni-Graft W SINUS; Braun, Melsungen, Germany), that closely resembled those of age-matched volunteers with a tendency towards more pronounced vortex formation. Another study performed with the same graft reported a more pronounced right-handed helical flow in the reconstructed aorta in comparison to non-operated volunteers (23). Those investigators did not encounter the vortices or re-circulating flow in the neo-root, even though different MRI parameters were applied (in particular a lower spatial resolution, $3.0 \times 1.7 \times 3.2$ vs. $\left.2.0 \times 2.0 \times 2.6 \mathrm{~mm}^{3}\right)(23)$.

Unlike previous studies, which reported single or small series of cases applying a semi-quantitative approach, our group has quantitatively characterize the intricate connection between the multiple morphological and dynamic features that determine the post-operative dynamics of aortic flow by analysing the complex set of data provided by 4D flow MRI.

Our initial results, obtained in a small population of 16 patients ( 8 with straight tube graft and 8 with reconstruction of SV), demonstrated vortex formation only in patients with reconstruction of SV during VSRR (12). Geometrical analysis of the aortic flow streamlines, based on the rotation angle with respect to the longitudinal axis of the vessel, revealed a significant increase of streamlines with high degree of rotation $\left(\varphi>270^{\circ}\right)$ at the border of root grafts in patients with a Valsalva graft $(10.3 \div 14.0 \%)$ compared to those with a straight tube $(2.2 \div 5.7 \%, \mathrm{P}=0.008)(12)$. This difference was associated with a reduction of wall shear stress at the bulb and sino-tubular junction (STJ) levels in patients with neo-SV reconstruction (12).

Interestingly, patients with straight tube grafts exhibit significantly more pronounced late systolic and early diastolic retrograde flow in the distal ascending aorta compared to volunteers or patients with a Valsalva graft (3). In the absence of significant aortic valve regurgitation, this retrograde flow may represent energy loss, which could be better preserved and redistributed in the grafts with neosinuses thanks to their reservoir function, resulting in a more gradual and continuous release during valve closing. However, some authors hypothesized that the reduced retrograde flow is due to the lesser elasticity of the Dacron graft, which compromised the physiological Windkessel effect introducing a highly localized increased stiffness in the ascending aorta (23).

The replacement of the native aortic wall with Dacron undoubtedly causes changes in vascular compliance, but these changes, in terms of aortic hemodynamics (helix flow, flow asymmetry, peak systolic velocities), are less pronounced after VSRR with recreation of neo-sinuses as compared to replacement with bioprosthetic valves (24).

Moreover, a significant increase in helicity downstream from the distal graft anastomosis has been demonstrated in patients with straight graft, as opposed to patients with a modified graft with recreation of neo-sinuses, which did not statistically differ from volunteers (3).

These findings imply a greater impact of the straight graft on the distal native aorta compared to the graft with neo-sinuses. However, further studies with larger populations are necessary.

\section{VSRR in bicuspid aortic valve (BAV)}

The BAV deserves specific consideration. The term bicuspid is an oversimplification of a spectrum of anatomical variations, and applying a single label was an historical error.

The Hannover School, led by Hans Joachim Schafers, had an extensive experience in reimplantation with BAV, which initially led to suboptimal results (25). As a result, BAV became a relative contraindication to VSRR. With further experience it became apparent that BAV had a higher incidence of leaflet abnormalities (26) that needed to be addressed in order to obtain satisfactory results. The invention of Schafer's Caliper, the introduction of precise measurements (coaptation level, lengths and height) and further standardization resulted improved outcomes in VSRR with BAVs nearly on par with trileaflet valves.

Early reports from small cohorts of BAV patients did not find significant differences in the aortic flow compared to patients with trileaflet valves after VSRR $(3,10)$ (Figure 3).

However, Semaan et al. (10) described some notsignificant differences in peak velocities and acceleration between patients with different valve anatomy (tricuspid and bicuspid valve) suggesting a contribution of the valve morphology to the hemodynamic outcome. Those authors observed eccentric flow patterns at the level of the aneurismal aortic root before VSRR, which normalized in both groups. Although the improvement in flow eccentricity 


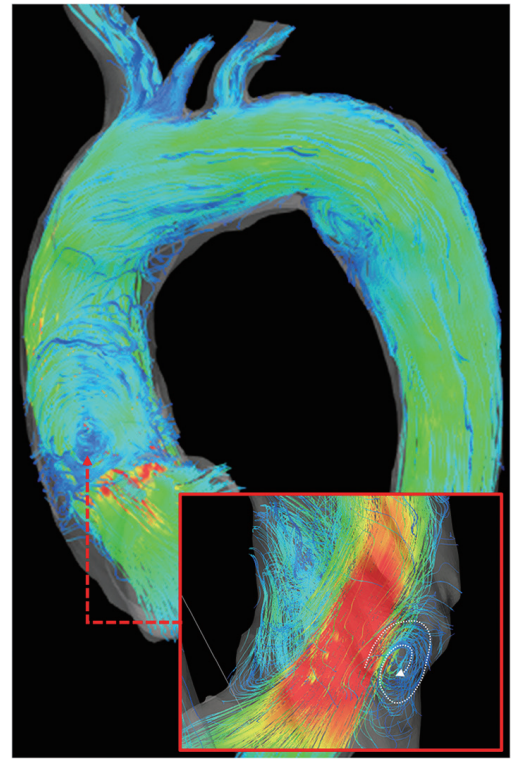

Figure 3 4D flow MRI images of a patient with bicuspid aortic valve who underwent VSRR with reconstruction of neo-sinuses. $3 \mathrm{D}$ streamline paths at peak systole delineate hemodynamics of bulk flow and confirm the generation of a large vortex (dotted curved arrows in the lower box) at the aortic root within the region of the sinuses of Valsalva, also seen in patients with anomalous valve morphology. MRI, magnetic resonance imaging.

in the proximal and mid-AAo was more pronounced in BAV subjects, it should be noted that valve repair was performed at the same time of VSRR surgery in more than half of the BAV cohort.

\section{Our institutional experience}

The Cornell International Consortium for Aortic Surgery (CICAS) arose from an international cooperation of two centers (Weill Cornell Medical Center in New York and European Hospital in Rome) with the aim of comparing different aortic surgery experiences and shedding light on the search for optimal surgical techniques.

Various software commercially available or under development enables $3 \mathrm{D}$ visualization of the instantaneous velocity field and the modifications of streamlines during the cardiac cycle and in the course of advancing the aortic flow from 4D flow MRI dataset. However, accurate image acquisition, processing and interpretation are still sophisticated skills that require close collaboration with imaging specialists.
The execution of MR examinations with high quality standards, with the adoption of identical imaging protocols on identical high field MR scanners, was made possible through collaboration with international reference centers for cardiovascular imaging (Weill Cornell Medicine in NYC and Sapienza University of Rome).

Finally, the accurate extrapolation of quantitative data from $4 \mathrm{D}$ flow MRI, which is essential for a rigorous methodology and in our experimental approach, required a centralized image lecture and very long time-consuming analysis by a team of experts (bioengineers) dedicated to MRI data processing (Politecnico di Milano) that has developed its own in-house software.

The close synergy of a multidisciplinary team consisting of members with very different but complementary skills (cardiac surgeons, cardiologists, radiologists, bioengineers) allows for the development of innovative methodological approaches aimed at responding to experimental questions and translating computational models into clinical benefits.

\section{Future perspectives}

Technological advances in the in vivo imaging of the impact of specific surgical techniques or grafts on fluid-dynamics will markedly transform the philosophy of cardiac surgery in the future. These new imaging techniques could change the practice of planning aortic replacement therapy, which will no longer only correct a pathological condition by restoring the normal anatomy but will also take into account which graft will result in optimal fluid-dynamics that best match the physiological requirements in the specific setting.

Further research interests of ours will be to investigate the interaction between specific graft designs with the mechanics of the valve leaflets and the possible implications on deterioration of valve function; the role of the vortices generated within the aortic root during systole in modulating coronary flow; the impact of graft shape on the formation of helical fluxes at the downstream aortic flow, including the potential impact on remodelling of the arch and descending aorta; or in determining the degree of stress on the sutures at the valvular annulus or the coronary arteries (Figure 4).

But as appetite comes with eating, so new frontiers of knowledge and new questions could be opened in light of new evidence that we hope will emerge from these studies. 

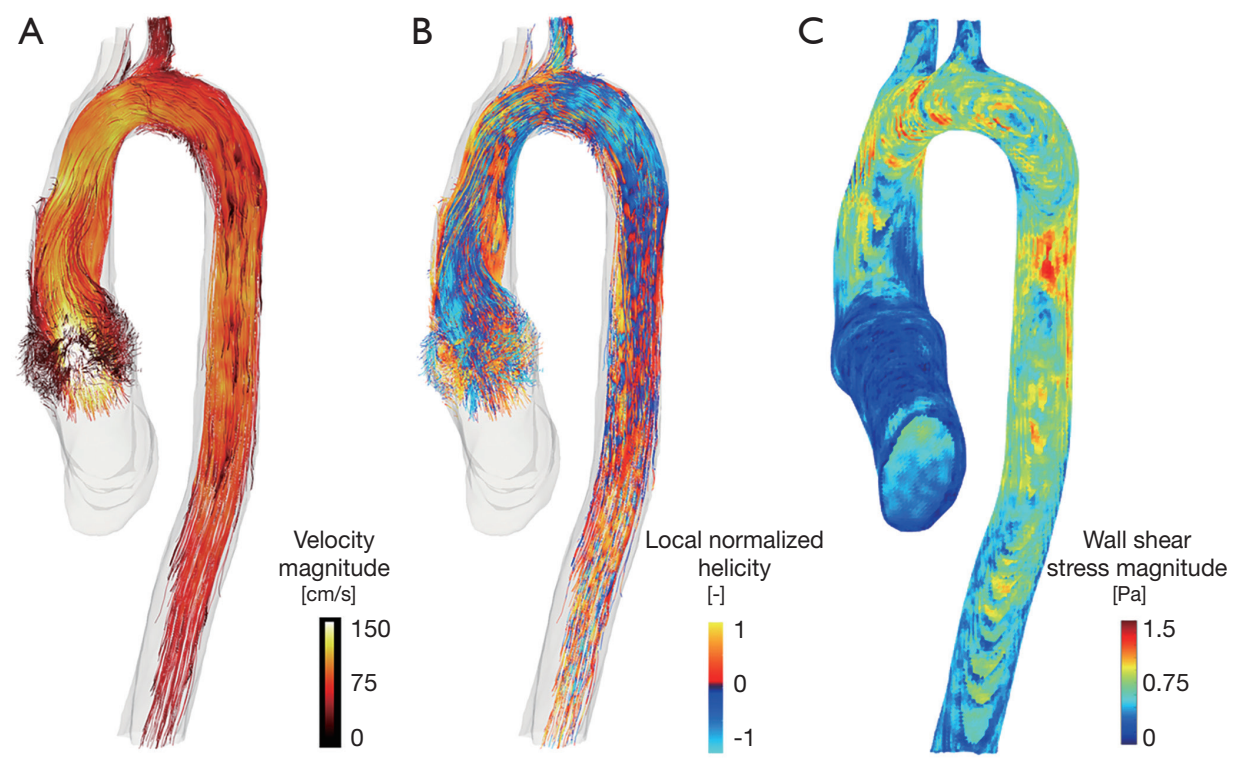

Figure 4 Visualization through 3D streamlines of the flow patterns within the aorta at peak systole, color-coded by (A) velocity magnitude and (B) local normalized helicity; (C) 3D distribution of the magnitude of wall shear stress vectors.

\section{Conclusions}

Multiple anatomical and functional aspects may affect aortic fluid dynamics after VSRR (including the native aortic morphology and the shape of the graft). 4D flow MRI is a unique tool for the in vivo analysis and visualization of blood-flow characteristics in patients after VSRR, by providing a comprehensive assessment of the $3 \mathrm{D}$ blood flow velocity fields and the anatomy of the entire thoracic aorta within a single acquisition. High hopes are placed in the possibility that this technique might in the future enable the early identification of those morphological or functional factors predisposing to complications or reveal the intricate connections between vortices within the sinuses of Valsalva, the valve closure mechanism and the modulation of coronary blood flow.

\section{Acknowledgements}

None.

\section{Footnote}

Conflicts of Interest: The manuscript has been presented at the Ninth Postgraduate Course on "Surgery of The Thoracic Aorta” in Bologna, Italy on November 13th-14th, 2017.

\section{References}

1. Srichai MB, Lim RP, Wong S, et al. Cardiovascular applications of phase-contrast MRI. AJR Am J Roentgenol 2009;192:662-75.

2. Markl M, Schnell S, Wu C, et al. Advanced flow MRI: emerging techniques and applications. Clin Radiol 2016;71:779-95.

3. Markl M, Draney MT, Miller DC, et al. Time-resolved three-dimensional magnetic resonance velocity mapping of aortic flow in healthy volunteers and patients after valvesparing aortic root replacement. J Thorac Cardiovasc Surg 2005;130:456-63.

4. Piatti F, Sturla F, Bissell MM, et al. 4D Flow Analysis of BAV-Related Fluid-Dynamic Alterations: Evidences of Wall Shear Stress Alterations in Absence of ClinicallyRelevant Aortic Anatomical Remodeling. Front Physiol 2017;8:441.

5. Yacoub MH, Gehle P, Chandrasekaran V, et al. Late results of a valve-preserving operation in patients with aneurysms of the ascending aorta and root. J Thorac Cardiovasc Surg 1998;115:1080-90.

6. David TE, Feindel CM. An aortic valve-sparing operation for patients with aortic incompetence and aneurysm of the ascending aorta. J Thorac Cardiovasc Surg 1992;103:61721; discussion 22.

7. Kvitting JP, Ebbers T, Wigstrom L, et al. Flow patterns in 
the aortic root and the aorta studied with time-resolved, 3-dimensional, phase-contrast magnetic resonance imaging: implications for aortic valve-sparing surgery. J Thorac Cardiovasc Surg 2004;127:1602-7.

8. Hope TA, Kvitting JP, Hope MD, et al. Evaluation of Marfan patients status post valve-sparing aortic root replacement with 4D flow. Magn Reson Imaging 2013;31:1479-84.

9. Oechtering TH, Frydrychowicz A, Sievers HH. Malrotated sinus vortices in straight graft valve-sparing aortic root treatment: A matter of concern? J Thorac Cardiovasc Surg 2017;154:794-7.

10. Semaan E, Markl M, Malaisrie SC, et al. Haemodynamic outcome at four-dimensional flow magnetic resonance imaging following valve-sparing aortic root replacement with tricuspid and bicuspid valve morphology. Eur J Cardiothorac Surg 2014;45:818-25.

11. Frydrychowicz A, Weigang E, Langer M, et al. Flowsensitive $3 \mathrm{D}$ magnetic resonance imaging reveals complex blood flow alterations in aortic Dacron graft repair. Interact Cardiovasc Thorac Surg 2006;5:340-2.

12. Galea N, Piatti F, Sturla F, et al. Novel insights by 4D flow imaging on aortic flow physiology after valve sparing root replacement with or without neo-sinuses. Interact Cardiovasc Thorac Surg 2018. [Epub ahead of print].

13. Erasmi A, Sievers HH, Scharfschwerdt M, et al. In vitro hydrodynamics, cusp-bending deformation, and root distensibility for different types of aortic valvesparing operations: remodeling, sinus prosthesis, and reimplantation. J Thorac Cardiovasc Surg 2005;130:1044-9.

14. Gaudino M, Girardi LN. What a great aortic surgeon would Messer Leonardo have been. J Thorac Cardiovasc Surg 2017;154:1219.

15. Bellhouse BJ, Bellhouse FH. Mechanism of closure of the aortic valve. Nature 1968;217:86-7.

16. De Paulis R, De Matteis GM, Nardi P, et al. A new aortic Dacron conduit for surgical treatment of aortic root pathology. Ital Heart J 2000;1:457-63.

doi: 10.21037/jovs.2018.03.17

Cite this article as: Galea N, Piatti F, Lau C, Sturla F, Weltert L, Carbone I, De Paulis R, Gaudino M, Girardi LN; The Cornell International Consortium for Aortic Surgery (CICAS). 4D flow characterization of aortic blood flow after valve sparing root reimplantation procedure. J Vis Surg 2018;4:95.
17. De Paulis R, De Matteis GM, Nardi P, et al. Analysis of valve motion after the reimplantation type of valve-sparing procedure (David I) with a new aortic root conduit. Ann Thorac Surg 2002;74:53-7.

18. Grande-Allen KJ, Cochran RP, Reinhall PG, et al. Recreation of sinuses is important for sparing the aortic valve: a finite element study. J Thorac Cardiovasc Surg 2000;119:753-63.

19. David TE, Armstrong S, Maganti M, et al. Long-term results of aortic valve-sparing operations in patients with Marfan syndrome. J Thorac Cardiovasc Surg 2009;138:859-64; discussion 63-4.

20. De Paulis R, Chirichilli I, Scaffa R, et al. Long-term results of the valve reimplantation technique using a graft with sinuses. J Thorac Cardiovasc Surg 2016;151:112-9.

21. Gaudino M, Di Franco A, Weltert L, et al. The role of neo-sinus reconstruction in aortic valve-sparing surgery. J Card Surg 2017;32:328-33.

22. Oechtering TH, Hons CF, Sieren M, et al. Time-resolved 3-dimensional magnetic resonance phase contrast imaging (4D Flow MRI) analysis of hemodynamics in valve-sparing aortic root repair with an anatomically shaped sinus prosthesis. J Thorac Cardiovasc Surg 2016;152:418-27.e1.

23. Frydrychowicz A, Berger A, Stalder AF, et al. Preliminary results by flow-sensitive magnetic resonance imaging after Tiron David I procedure with an anatomically shaped ascending aortic graft. Interact Cardiovasc Thorac Surg 2009;9:155-8.

24. Collins JD, Semaan E, Barker A, et al. Comparison of Hemodynamics After Aortic Root Replacement Using Valve-Sparing or Bioprosthetic Valved Conduit. Ann Thorac Surg 2015;100:1556-62.

25. Aicher D, Langer F, Lausberg H, et al. Aortic root remodeling: ten-year experience with 274 patients. J Thorac Cardiovasc Surg 2007;134:909-15.

26. Aicher D, Kunihara T, Abou Issa O, et al. Valve configuration determines long-term results after repair of the bicuspid aortic valve. Circulation 2011;123:178-85. 


\section{Supplementary material}

Particle paths of patients with straight tube graft (Figure S1) and graft with reconstruction of neo-sinuses (Figure S2). The color of the streamlines represents the flow velocity. In both cases it is well depicted the difference between the central high-speed laminar flow (orange) and the peripheral slow turbulent flow (blue), that is irregular and chaotic in the straight graft (Figure S1) and organized in vortexes in the neo-sinuses graft (Figure S2).

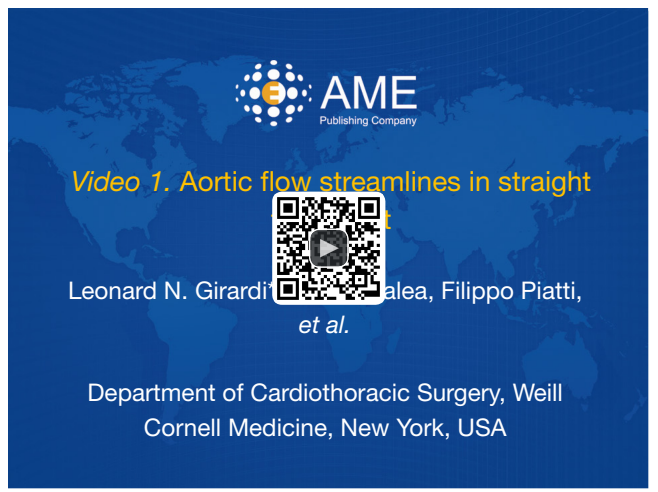

Figure S1 Aortic flow streamlines in straight tube graft (27). Available online: http://www.asvide.com/article/view/24676

\section{References}

27. Galea N, Piatti F, Lau C, et al. Aortic flow streamlines in straight tube graft. Asvide 2018;5:506. Available online: http:// www.asvide.com/article/view/24676

28. Galea N, Piatti F, Lau C, et al. Aortic flow streamlines in graft with reconstruction of neo-sinuses. Asvide 2018;5:507. Available online: http://www.asvide.com/article/view/24677

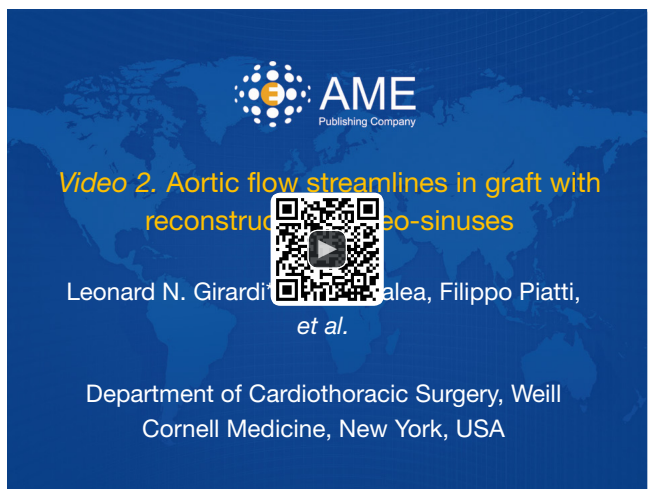

Figure S2 Aortic flow streamlines in graft with reconstruction of neo-sinuses (28).

Available online: http://www.asvide.com/article/view/24677 